\title{
Comparison of Healthcare Utilization and Life-Sustaining Interventions between Elderly Patients with Dementia and those with Cancer near the End of Life: A Nationwide, Population-Based Study in Taiwan
}

Short title: End-of-life care for dementia in Taiwan

Yu-Han Chen ${ }^{1,2}$, Chung-Han $\mathrm{Ho}^{3,4}$, Chien-Cheng Huang ${ }^{5,6,7,8}$, Ya-Wen $\mathrm{Hsu}^{3,4}$, Yueh-Chun Chen ${ }^{1,9}$, Ping-Jen Chen ${ }^{1,6,8, *}$, Guan-Ting Chen ${ }^{1,10}$, Jhi-Joung Wang ${ }^{3,10}$

${ }^{1}$ Palliative Care Center, Chi-Mei Medical Center, Tainan, Taiwan

${ }^{2}$ Department of Family Medicine, Chi-Mei Medical Center, Tainan, Taiwan

${ }^{3}$ Department of Medical Research, Chi-Mei Medical Center, Tainan, Taiwan

${ }^{4}$ Department of Hospital and Health Care Administration, Chia Nan University of Pharmacy and Science, Tainan, Taiwan

${ }^{5}$ Department of Emergency Medicine, Chi-Mei Medical Center, Tainan, Taiwan

${ }^{6}$ Department of Geriatrics and Gerontology, Chi-Mei Medical Center, Tainan, Taiwan

${ }^{7}$ Department of Environmental and Occupational Health, College of Medicine National Cheng Kung University, Tainan, Taiwan

${ }^{8}$ Bachlor Program of Senior Services, Southern Taiwan University of Science and Technology, Tainan, Taiwan

${ }^{9}$ Department of Nursing, Chi-Mei Medical Center, Tainan, Taiwan

${ }^{10}$ Department of Anesthesiology, Chi-Mei Medical Center, Tainan, Taiwan

\section{* Corresponding Author:}


Ping-Jen Chen, MD, Palliative Care Center and Department of Geriatrics and Gerontology, Chi-Mei Medical Center, 901 Zhong-hua Road, Yong-kang Dist., Tainan City 710, Taiwan

Tel: +886-6-281-2811 ext. 57199, Fax: +886-6-283-3806; Email:

pingjen.chen@gmail.com

Word counts: abstract: 200; full article: 2722; tables: 4; figure:1 


\section{Abstract}

Aim: Little is known about the pattern of healthcare services for end-of-life patients with dementia (PwDs) in East Asia. We compared this pattern between PwDs and cancer patients in their last year of life in Taiwan.

Methods: Taiwan's National Health Insurance Research Database was applied for this case-control analysis. The records of patients who had dementia and died between 2002 and 2011 were reviewed. The control group was decedents with cancer. The utilization of hospitalization, emergency department visits, and life-sustaining interventions during the last year of life between two groups were compared.

Results: Of the 2724 patients enrolled, 908 had dementia and 1816 had cancer. PwDs were more likely to have higher frequency of admission to hospital and ICU and longer stays by comparing to cancer patients. PwDs had a higher risk of enteral tube insertion and feeding, endotracheal intubation and tracheostomy, mechanical ventilation, hemodialysis, and cardiopulmonary resuscitation $(\mathrm{OR}=4.36,95 \% \mathrm{CI}=3.51-5.41)$ the highest among selected procedures.

Conclusions: PwDs in their last year of life in Taiwan underwent aggressive interventions significantly more frequently than did their counterparts in Western countries. Providing comfort-centered care for better quality-of-life for end-of-life PwDs is a priority of Taiwan's national health policy.

Key words: cancer, dementia, end-of-life, life-sustaining treatment, utilization 


\section{Introduction}

Dementia is a neurodegenerative illness and one of the major causes of disability and dependency among the elderly. Patients with dementia (PwDs) require a great deal of healthcare resources throughout the course of the disease. ${ }^{1}$ A 1-year follow-up study in Taiwan indicated s that PwDs had significantly more outpatient visits and hospitalization days than did those without dementia. ${ }^{2}$

In Western countries, comparisons of healthcare resource utilization between PwDs and cancer patients near the end of life are common because that these two diseases represent different illness trajectories. ${ }^{3,4}$ Previous studies, however, have documented various patterns. One study ${ }^{5}$ reported that, during the last month of life, PwDs in acute hospitals and long-term care facilities (LTCFs) are given less aggressive therapy than are patients without dementia. Other studies ${ }^{3,6}$ have shown that patients with advanced dementia commonly undergo more hospital transfers and burdensome interventions (e.g., tube feeding) than do patients with terminal cancer near the end of life.

There are cross-cultural differences between Western and Asian countries that might influence medical professionals' and caregivers' attitudes toward terminally ill patients. One international study ${ }^{7}$ reported that one-third of Japanese physicians in intensive care units (ICUs) would not apply do-not-resuscitate (DNR) orders to patients with anoxic encephalopathy and in a vegetative state. When life-threatening events occurred in these vegetative patients, Japanese physicians chose aggressive interventions with continued full support, while physicians from Western countries would be more commonly withdraw life-sustaining treatments. Advanced dementia is generally not perceived as a terminal condition, and life preservation is the prevailing 
value in Asian societies like China, India, Israel, and Japan. ${ }^{8-11}$ A lack of discussion about patients' advance directives also causes a family's surrogate decision-making to be the predominant factor of end-of-life management. Furthermore, palliative care, including opioids for symptom relief, might be limited for PwDs in the national healthcare systems in Asian countries. ${ }^{11}$

Little is known about using aggressive medical treatments for PwDs and cancer patients near the end of their life in East Asia. We compared the utilization of healthcare services and life-sustaining interventions for PwDs and cancer patients in Taiwan in their last year before death. We hypothesized that PwDs undergo more aggressive procedures than those with cancer near the end of their life.

\section{Methods}

\section{Data source and study population}

All data are from the Taiwan National Health Insurance Research Database (NHIRD). The NHI is a universal single-payer scheme that covers $99.9 \%$ of Taiwan's legal residents. From the NHIRD, we obtained the Longitudinal Health Insurance Database (LHID2000), which contains all the original claims data of 1,000,000 enrollees randomly sampled since 2000. Because the patient data are deidentified, the confidentiality of personal information in this database is assured, and using the data must be authorized by the Taiwan Bureau of National Health Insurance (BNHI). Our research protocol was approved by the institutional review board of Chi-Mei Medical Center.

The study population included patients $\geq 18$ years old who had been diagnosed with dementia and died during 2002-2011. Decedents with a diagnosis of dementia were 
selected using the following criteria: (i) At least three outpatient service claims with the International Classification of Diseases, Ninth Revision, Clinical Modification (ICD-9-CM) codes of dementia (290.1x-209.4x, 291.2, 292.82, 294.1x, 294.8, 331.0, $331.1 x, 331.2,331.82$ ) at any clinic within one year after the first dementia code; (ii) any single hospitalization for dementia among the 5 principal claims diagnosis codes. We identified a control group of decedents with cancer who had catastrophic certificates with ICD-9-CM cancer codes: 140-208 (the detail was shown in Supplementary Table 1). Patients who met the criteria for both dementia and cancer were excluded.

\section{Measurements}

We identified 1072 PwDs and 13,771 control patients who met the eligibility criteria. 908 PwDs were matched to two controls each $(n=1816)$ by age and gender $(\mathrm{N}=2724)$. The characteristics among subjects excluded was shown in Supplementary Table 2. We measured the utilization of healthcare services during the last year of life between these two groups by comparing the frequency of hospital admissions, length of inpatient stays, emergency department (ED) visits, and non-palliative interventions (enteral tube insertion and feeding, blood transfusion, respiratory therapies, hemodialysis, and cardiopulmonary life-sustaining treatments).

The comorbidities were also classified using ICD-9-CM codes: diabetes mellitus (250), cerebrovascular disease (CVD) (430-438), heart failure (428), chronic obstructive pulmonary disease (COPD) (491.2x, 492, 494, 496), liver cirrhosis $(571.2,571.5)$, renal failure $(585.4-6,586)$, and ischemic heart disease/coronary artery disease (CAD) (410-414).

\section{Statistical analysis}

Student's $t$ test or the Wilcoxon rank-sum test for continuous variables, and 
Pearson's $\chi^{2}$ test for categorical variables were used to compare differences between patients who died of dementia and those who died of cancer. The association between matched cases and controls was measured using a conditional logistic regression model to calculate the odds ratios (ORs) and 95\% confidence intervals (CIs). Significance was set at $\mathrm{p}<0.05$. SAS 9.4 for Windows (SAS Institute, Inc., Cary, NC) was used for all statistical analyses.

\section{Results}

\section{Characteristics of the study group}

We registered 2,724 decedents in this study. PwDs had a significantly $(\mathrm{p}<0.001$ for all) higher proportion than did patients with cancer of diabetes, cerebrovascular disease, and renal failure before death (Table 1).

\section{Utilization of healthcare services}

In the last year before death, the most common causes of hospitalization and ED visits by dementia group were infectious diseases and their related complications (Table 2). Chemotherapy was the major cause of hospitalization in the cancer group. After excluding the diagnoses related to cancer therapy, the common causes of hospitalization and ED visits in the cancer group were also infectious diseases.

PwDs had higher proportion of ICU admissions than did cancer patients (odds ratio $[\mathrm{OR}]=3.45 ; 95 \%$ confidence interval $[\mathrm{CI}]=2.90-4.11)$ (Table 3). Among patients who had hospitalization, PwDs were more likely to have high frequency of admission to the hospital $(\mathrm{OR}=1.26 ; 95 \% \mathrm{CI}=1.04-1.52)$ and $\mathrm{ICU}(\mathrm{OR}=2.01,95 \% \mathrm{CI}=1.49-2.70)$, and to have longer hospital $(\mathrm{OR}=1.40 ; 95 \% \mathrm{CI}=1.16-1.69)$ and $\mathrm{ICU}(\mathrm{OR}=1.92 ; 95 \%$ $\mathrm{CI}=1.41-2.61)$ stays, by comparing to cancer patients. 


\section{Life-sustaining treatments}

The prevalence of using life-sustaining treatments, except for blood transfusions, was significantly $(\mathrm{p}<0.001)$ higher in PwDs (Table 4). After confounding covariates had been adjusted for, PwDs were more likely to undergo aggressive interventions. Cardiopulmonary resuscitation $(\mathrm{CPR})$ had the highest risk $(\mathrm{OR}=4.36 ; 95 \% \mathrm{CI}=$ 3.51-5.41). The use of CPR significantly increased within one month before death in two groups (Figure 1), and the frequency of CPR was more than 3 times higher for PwDs than for cancer patients.

\section{Discussion}

Our nationwide, population-based study found that, in Taiwan, PwDs in their last year of life are at a higher risk of undergoing aggressive life-sustaining interventions than are patients with cancer. To our knowledge, this is one of the pioneer nationwide investigations of healthcare utilization for PwDs near the end of life in East Asia.

\section{Comparison with international data}

Other studies have reported that burdensome hospitalizations had limited clinical benefits for cognitively impaired nursing-home residents. Gozalo et al. ${ }^{1}$ found that $19 \%$ of nursing-home residents had at least one burdensome transition near the end of life, and that $9.1 \%$ with advanced cognitive impairment were admitted to the ICU during their last 30 days of life. In Hong Kong, a longitudinal cohort study ${ }^{12}$ found that elderly patients with advanced cognitive impairment in LTCFs had a mean frequency of about 3.1 ED visits and 2.7 acute hospital admissions during the 12 months of follow-up ${ }^{12}$. We found that $48.57 \%$ of PwDs in Taiwan had three or more acute hospital admissions and $20.48 \%$ had two or more ICU admissions during their last year of life. 
All the procedures of medical treatments, including feeding tube insertion for patients, whether they are admitted into acute hospitals or live in LTCFs, are reimbursed by the NHI in Taiwan. A retrospective study ${ }^{13}$ of LTCFs in Italy reported that $20.5 \%$ of PwDs were fed with a nasogastric (NG) tube or a percutaneous endoscopic gastrostomy (PEG) in their last 30 days of life. North American studies ${ }^{3,10}$ reported that $25 \%$ of patients in the US with advanced dementia were tube-fed near their end of life ${ }^{3}$, and that $11 \%$ of patients in Canada with end-stage dementia were tube-fed in hospitals ${ }^{10}$. In Asia, $66 \%$ of nursing-home residents in Hong Kong with advanced cognitive impairment were enterally fed in their last year of life ${ }^{12}$; whereas tube-feeding rate among patients with advanced dementia in Israel is $52.9 \%{ }^{10}$. Compared with findings from international studies, the prevalence of tube feeding in the PwDs in our study (67.4\%) significantly different from the rates in Europe and North America, and were higher than in most other Asian studies. The adjusted odds ratio of tube feeding between the PwD group and the cancer group in our study was 2.75 , significantly higher than the 2.21 in a US study ${ }^{3}$.

One retrospective US analysis ${ }^{5}$ reported that only $13 \%$ of its PwDs underwent mechanical ventilation and that $3.9 \%$ of them underwent hemodialysis in the last 30 days of life, but in our survey, $61.45 \%$ of the PwDs underwent mechanical ventilation, and $17.62 \%$ underwent hemodialysis. The CPR rate in our dementia group (33.92\%) was much higher than that in the data reported in an Italian study ${ }^{13}(2 \%)$. In the present study, the frequency of CPR near the end-of-life for PwDs was much higher than that for cancer patients, which implies that PwDs tend to continually undergo life-sustaining treatments when they are critically ill. Although the mortality rates of PwDs increase with the severity of cognitive impairment ${ }^{14}$, results of a large-scale population-based 
cohort study ${ }^{15}$ across China, India, and Latin America showed that more than half of the mortality occurred in people with mild dementia at baseline, which means that, in our survey, resuscitation and additional treatments might have been done to reverse acute, unexpected physical conditions such as pneumonia and septicemia (see Supplementary Tables 3, 4), and to prevent mortality in PwDs, higher proportion of whom were probably in an mild or moderate rather than an advanced stage of dementia. However, the survival outcomes of these frail patients with prevalent comorbidities might not be as good as expected by medical professionals. In addition, aggressive interventions might be burdensome for patients and families.

The discrepancy between our results in an Asian country and data from Western countries might be explained by the combination of culture, religion, administrative financial incentives, and the doctor-family-patient relationship ${ }^{8-11,16,17}$. An investigation ${ }^{9}$ that explored different methods of caring for PwDs in the Netherlands and India reported that the PwDs themselves in India were rarely involved in deciding what kind of end-of-life care they should be given and underwent many aggressive treatments to prolong their life. PwDs were detected earlier in the Netherlands, and advance directives were more frequently established by patients, family, and healthcare professionals via a "dialogical" process, which led to less life-sustaining management in medically futile conditions. A study ${ }^{10}$ that compared tube-feeding for end-stage dementia across Canada and Israel reported that because of religion and culture, Israel's administrative guidelines favored using NG tubes to save and extend life, which led to a huge gap in tube-feeding prevalences between Canada and Israel.

Chinese culture, which is predominantly Confucian, is the main social context in Taiwan. Most of the Chinese elderly refrain from discussing death-related topics, which 
makes advance end-of-life care planning difficult ${ }^{18}$. Even for PwDs who had expressed their preferences for end-of-life decisions, the proportion of discrepancy between patients and their caregivers was $48.3 \%$ for tube feeding, $48.5 \%$ for CPR, and $60.3 \%$ for mechanical ventilation in a Taiwanese investigation ${ }^{19}$. A majority of medical professionals and patients relied more on families' preferences and physician authority than on patient autonomy ${ }^{17,18}$. Because it is a Chinese cultural belief that dying relatives become "hungry ghosts" if they are not supplied with artificial nutrition until they $\operatorname{die}^{20}$, and because the general public had insufficient understanding of life-sustaining treatments $^{8}$, Chinese family caregivers are usually reluctant to prevent aggressive interventions.

In contrast, one American study ${ }^{21}$ reported that using intensive procedures during the end of life was strongly affected by individual characteristics and regional practice patterns. NHI makes hospitalization and ICU care easily available to and affordable for almost everyone in Taiwan. The fee-for-service payment mechanisms in NHI schemes in Taiwan might be incentives for the overutilization of medical services here, however ${ }^{22}$. Furthermore, medical dispute cases in Taiwan have significantly increased over the past two decades. Almost $80 \%$ are criminal suits, which might cause medical professionals to practice defensive medicine by doing additional and aggressive life-prolonging interventions even for patients with poor prognoses ${ }^{23}$.

\section{Benefits and barriers of giving PwDs comfort-centered care}

Several studies ${ }^{24-26}$ have shown that palliative care improved the quality of end-of-life care and increased patient satisfaction, and reduced, for cancer and non-cancer patients, the use of aggressive medical services: ICU admission, artificial nutrition, and artificial hydration. One American study ${ }^{27}$ reported that interviewed 
bereaved family members of deceased PwDs said that hospice services improved their perceptions of the quality of care for the patients and the quality of the dying experience $^{27}$. However, providing hospice services to PwDs is still limited in Taiwan: less than $0.1 \%$ of all patients were given in-patient hospice services in $2013^{28}$.

There might be some barriers for giving palliative care to PwDs near the end of life. First, advanced dementia is not normally considered a progressively terminal illness by the general population or medical professionals in many Asian countries ${ }^{8-11,29}$. Second, the "dying trajectories" of cancer and dementia are quite different. While the gradual deterioration of people with dementia for a longer time is recognized, the trajectory might be cut short by death after an unexpected acute illness like pneumonia ${ }^{3,4,15}$. It is challenging to accurately estimate life expectancy in advanced dementia and provide timely palliative care based on a paradigm of survival prediction ${ }^{30}$. Early discussion of care planning with the PwDs and their family members about acceptable life-sustaining measures is urgently needed ${ }^{19,21}$.

Neurologists are the main specialists for treating PwDs in Taiwan. Internal medicine physicians, however, usually take the major responsibility for inpatient care of PwDs in their late stage of life. The clinical interpretation, therefore, suggests that both neurologists and internists should upgrade their knowledge and skills for providing comfort-oriented care, and for helping PwDs and their relatives make decisions in the patients' best interests.

\section{Strengths and limitations}

The strengths of the present study include its long period of case registration, its age- and gender-matched compared groups, and its using a nationwide database.

Dementia is a major health economic priority worldwide, yet the evidence base is small. 
The present study provides a nationwide pattern of clinical practice.

The limitations are that the study was done only in Taiwan, a small country in Asia, which limits its generalizability to other areas. Taiwan's cultural values about disease awareness and treatment choice, however, are strongly influenced by Chinese and Japanese values, so that our results gave possible hints about the condition in other nearby Asian countries. Second, the limitation of major diagnostic codes can be identified in outpatient service claims, and insufficient awareness among healthcare professionals in hospitals of coding dementia as a crucial underlying disease for patients, might have caused us to underestimate the prevalence of dementia in our population-based study. Another limitation is that the atypical presentation of diseases in the elderly and under-reported symptoms both contribute to an underestimation of cancer in PwDs, which lead to a possible selection bias. Finally, the severity of disease cannot be identified and classified because the NHIRD does not provide that information.

The noteworthy high risk of PwDs undergoing life-sustaining treatments in their last year of life, regardless of the level of their cognitive impairment, indicates that they are not be given sufficient palliative care. A national health policy that endorses palliative care for PwDs near the end of life and continuous end-of-life care education for multidisciplinary medical professionals should be advocated. Additional studies are needed to better understand the mechanisms and key determinants underlying the use of life-sustaining treatments in patients with different levels of dementia, and to evaluate the quality of end-of-life care.

\section{Acknowledgments}


This study is based in part on data from the Taiwan National Health Insurance Research Database provided by the National Health Insurance Administration, Ministry of Health and Welfare, and managed by the National Health Research Institutes. We thank Bill Franke for his English editing.

\section{Disclosure statement}

The authors declare that there are no conflicts of interests regarding the publication of this paper. 


\section{References}

1. Gozalo P, Teno JM, Mitchell SL, Skinner J, Bynum J, Tyler D. End-of-life transitions among nursing home residents with cognitive issues. $N$ Engl J Med 2011; 365(13): 1212-1221.

2. Chung SD, Liu SP, Sheu JJ, Lin CC, Lin HC, Chen CH. Increased healthcare service utilizations for patients with dementia: a population-based study. PLoS One 2014; 9(8): e105789.

3. Mitchell SL, Kiely DK, Hamel MB. Dying with advanced dementia in the nursing home. Arch Intern Med 2004; 164: 321-326.

4. Barclay S, Froggatt K, Crang C, Mathie E, Handley M, Iliffe S et al. Living in uncertain times: trajectories to death in residential care homes. Br J Gen Pract 2014; 64(626): e576-583.

5. Richardson SS, Sullivan G, Hill A, Yu W. Use of aggressive medical treatments near the end of life: differences between patients with and without dementia. Health Serv Res 2007; 42(1 Pt 1): 183-200.

6. Goldfeld KS, Stevenson DG, Hamel MB, Mitchell SL. Medicare expenditures among nursing home residents with advanced dementia. Arch Intern Med 2011 171(9): 824-830.

7. Yaguchi A, Truog RD, Curtis JR, Luce JM, Levy MM, Melot C et al. International differences in end-of-life attitudes in the intensive care unit: results of a survey. Arch Intern Med 2005; 165(17): 1970-1975.

8. Kwok T, Twinn S, Yan E. The attitudes of Chinese family caregivers of older people with dementia towards life sustaining treatments. J Adv Nurs 2007; 58(3): 256-262.

9. Sowmini CV, Vries RD. A cross cultural review of the ethical issues in dementia care in Kerala, India and The Netherlands. Int J Geriatr Psychiatry 2009; 24(4): 329-334.

10. Clarfield AM, Monette J, Bergman H, Monette M, Ben-Israel Y, Caine Y et al. Enteral feeding in end-stage dementia: a comparison of religious, ethnic, and national differences in Canada and Israel. J Gerontol A Biol Sci Med Sci 2006; 61(6): 621-627.

11. van der Steen JT, Hertogh CM, de Graas T, Nakanishi M, Toscani F, Arcand M. Translation and cross-cultural adaptation of a family booklet on comfort care in dementia: sensitive topics revised before implementation. J Med Ethics 2013; 39(2): 104-109.

12. Luk JK, Chan WK, Ng WC, Chiu PK, C H, Chan TC et al. Mortality and health services utilisation among older people with advanced cognitive impairment living in residential care homes. Hong Kong Med J 2013; 19(6): 518-524.

13. Di Giulio P, Toscani F, Villani D, Brunelli C, Gentile S, Spadin P. Dying with advanced dementia in long-term care geriatric institutions: a retrospective study. J Palliat Med 2008; 11(7): 1023-1028.

14. Guehne U, Riedel-Heller S, Angermeyer MC. Mortality in dementia. Neuroepidemiology 2005; 25(3): 153-162.

15. Prince M, Acosta D, Ferri CP, Guerra M, Huang Y, Llibre Rodriguez JJ et al. Dementia incidence and mortality in middle-income countries, and associations with indicators of cognitive reserve: a 10/66 Dementia Research Group 
population-based cohort study. Lancet 2012; 380(9836): 50-58.

16. Phua J, Kee AC, Tan A, Mukhopadhyay A, See KC, Aung NW et al. End-of-life care in the general wards of a Singaporean hospital: an Asian perspective. $J$ Palliat Med 2011; 14(12): 1296-1301.

17. Cong Y. Doctor-family-patient relationship: the Chinese paradigm of informed consent. J Med Philos 2004; 29(2): 149-178.

18. Lee HT, Cheng SC, Dai YT, Chang M, Hu WY. Cultural perspectives of older nursing home residents regarding signing their own DNR directives in Eastern Taiwan: a qualitative pilot study. BMC Palliat Care 2016; 15: 45.

19. Tsai CF, Lee YT, Lee WJ, Hwang JP, Wang SJ, Fuh JL. Depression of family caregivers is associated with disagreements on life-sustaining preferences for treating patients with dementia. 2015; 10(7): e0133711.

20. Rifkin DE. Feeding the hungry ghosts. Am J Kidney Dis 2010; 55(1): A33-A34.

21. Tschirhart EC, Du Q, Kelley AS. Factors influencing the use of intensive procedures at the end of life. J Am Geriatr Soc 2014; 62(11): 2088-2094.

22. Waters HR, Hussey P. Pricing health services for purchasers--a review of methods and experiences. Health Policy 2004; 70(2): 175-184.

23. Wu CY, Weng HC, Chen RC. Time trends of assessments for medical dispute cases in Taiwan: a 20-year nationwide study. Intern Med J 2013; 43(9): 1023-1030.

24. Temel J, Greer J, Muzikansky A, Gallagher E, Admane S, Jackson V et al. Early palliative care for patients with metastatic non-small-cell lung cancer. $N$ Engl $J$ Med 2010; 363: 733-742.

25. Brumley R, Enguidanos S, Jamison P, Seitz R, Morgenstern N, Saito S et al. Increased satisfaction with care and lower costs: results of a randomized trial of in-home palliative care. J Am Geriatr Soc 2007; 55: 993-1000.

26. Miller S, Lima J, Susan LM. Influence of hospice on nursing home residents with advanced dementia who received Medicare-skilled nursing facility care near the end of life. J Am Geriatr Soc 2012; 60(11): 2035-2041.

27. Teno JM, Gozalo PL, Lee IC, Kuo S, Spence C, Connor SR. Does hospice improve quality of care for persons dying from dementia? J Am Geriatr Soc 2011; 59(8): 1531-1536.

28. BNHI. Ministry of Health and Welfare. 2013 National Health Insurance Annual Statistical Report. Taipei: Bureau of National Health Insurance. 2014. Available in English at:

<http://www.mohw.gov.tw/EN/Ministry/Statistic.aspx?f_list_no=474\&fod_list_no=523 6>. Accessed 15 February 2017.

29. Pang M, Volicer L, Chung P, Chung Y, Leung W, White P. Comparing the ethical challenges of forgoing tube feeding in American and Hong Kong patients with advanced dementia. J Nutr Health Aging 2007; 11(6): 495-501.

30. Mitchell SL, Miller SC, Teno JM, Kiely DK, Davis RB, Shaffer ML. Prediction of 6-month survival of nursing home residents with advanced dementia using ADEPT vs hospice eligibility guidelines. JAMA. 2010; 304(17): 1929-1935. 


\section{Figure Legend}

Figure 1. Frequency of cardiopulmonary resuscitation in the dementia group and the cancer group within the last one year of life

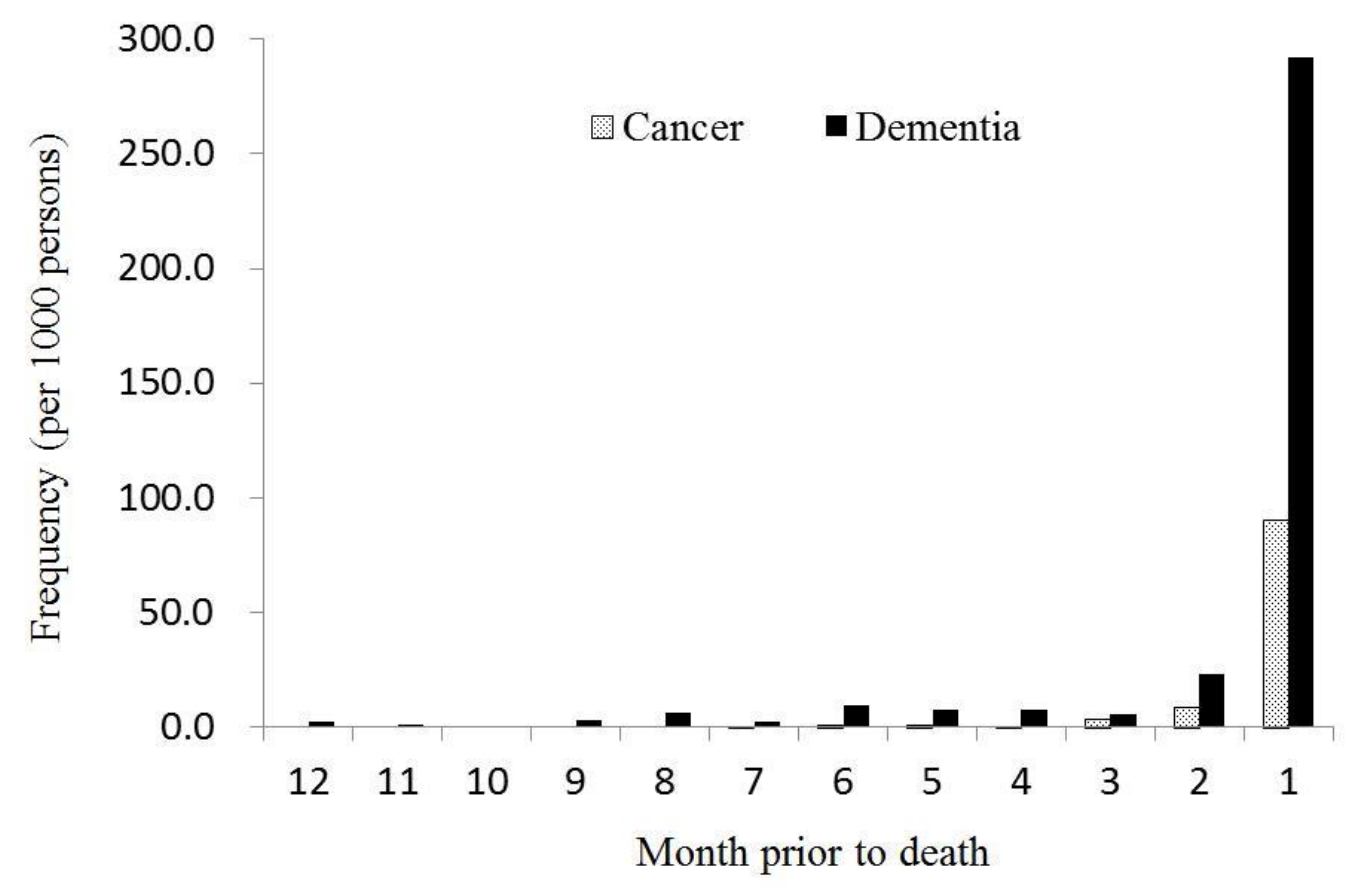




\section{Supplementary Information Legend}

Supplementary Table 1. Top 10 categories of patients diagnosed with cancer

Supplementary Table 2. The characteristics among subjects excluded in dementia and cancer group $(\mathrm{N}=12119)$

Supplement Table 3. TOP 5 major diagnoses of diseases of hospitalization and emergency department visits that cardiopulmonary resuscitation (CPR) was performed during the period of stay for patients with dementia and those with cancer in the last year of life

Supplement Table 4. TOP 5 major diagnoses of diseases of hospitalization and emergency department visits that endotracheal intubation was performed during the period of stay for patients with dementia and those with cancer in the last year of life 
Table 1. Demographic characteristics of the study participants: patients with dementia and patients with cancer $(\mathrm{N}=2724)$

\begin{tabular}{|c|c|c|c|}
\hline $\begin{array}{c}\text { Group: } \\
\text { Variables } \\
\end{array}$ & $\begin{array}{l}\text { Dementia } \\
(\mathrm{n}=908) \\
\end{array}$ & $\begin{array}{c}\text { Cancer } \\
(\mathrm{n}=1816) \\
\end{array}$ & p-value \\
\hline Age at death, mean \pm SD & $77.12 \pm 11.26$ & $77.12 \pm 11.25$ & 0.9936 \\
\hline \multicolumn{4}{|l|}{ Age at death, n (\%) } \\
\hline$<50$ & $34(3.74)$ & $68(3.74)$ & 1.0000 \\
\hline $50-65$ & $70(7.71)$ & $141(7.76)$ & \\
\hline $65-80$ & $374(41.19)$ & $747(41.13)$ & \\
\hline$\geq 80$ & $430(47.36)$ & $860(47.36)$ & \\
\hline \multicolumn{4}{|l|}{ Gender, n $(\%)$} \\
\hline Male & $522(57.49)$ & $1044(57.49)$ & 1.0000 \\
\hline \multicolumn{4}{|l|}{ Comorbidities, n (\%) } \\
\hline Diabetes & $274(30.18)$ & $421(23.18)$ & $<0.001$ \\
\hline Cerebrovascular disease & $358(39.43)$ & $280(15.42)$ & $<0.001$ \\
\hline Heart failure & $97(10.68)$ & $156(8.59)$ & 0.0761 \\
\hline Chronic obstructive pulmonary disease & $152(16.74)$ & $283(15.58)$ & 0.4373 \\
\hline Liver cirrhosis & $28(3.08)$ & $123(6.77)$ & $<0.001$ \\
\hline Renal failure & $41(4.52)$ & $31(1.71)$ & $<0.001$ \\
\hline Coronary artery disease & $167(18.39)$ & $384(21.15)$ & 0.0917 \\
\hline
\end{tabular}

$\mathrm{SD}=$ standard deviation 
Table 2. Common causes of hospitalization and emergency department visits in the last year of life by patients with dementia and patients with cancer

\begin{tabular}{|c|c|c|c|c|}
\hline & Group: & Dementia & & Cancer \\
\hline Rank & Diagnosis & $\mathbf{n}(\%)^{\dagger}$ & Diagnosis $^{\dagger}$ & n $(\%)$ \\
\hline 1 & Pneumonia and pneumonitis & $\begin{array}{l}574 \\
(13.62)\end{array}$ & $\begin{array}{l}\text { Pneumonia and } \\
\text { pneumonitis }\end{array}$ & $352(4.43)$ \\
\hline 2 & Acute respiratory failure & $\begin{array}{l}515 \\
(12.22)\end{array}$ & Sepsis & $168(2.11)$ \\
\hline 3 & Urinary tract infection & $193(4.58)$ & Urinary tract infection & $167(2.10)$ \\
\hline 4 & Sepsis & $192(4.56)$ & $\begin{array}{l}\text { Acute respiratory } \\
\text { failure }\end{array}$ & $159(2.00)$ \\
\hline 5 & $\begin{array}{l}\text { Obstructive chronic bronchitis } \\
\text { with acute exacerbation }\end{array}$ & $146(3.46)$ & Fever & $117(1.47)$ \\
\hline 6 & Chronic renal failure & $122(2.90)$ & Liver cirrhosis & $96(1.21)$ \\
\hline 7 & Chronic respiratory failure & $116(2.75)$ & $\begin{array}{l}\text { Gastrointestinal tract } \\
\text { hemorrhage }\end{array}$ & $91(1.14)$ \\
\hline 8 & Fever & $100(2.37)$ & Abdominal pain & $67(0.84)$ \\
\hline 9 & $\begin{array}{l}\text { Gastrointestinal tract } \\
\text { hemorrhage }\end{array}$ & $63(1.50)$ & $\begin{array}{l}\text { Congestive heart } \\
\text { failure }\end{array}$ & $56(0.70)$ \\
\hline 10 & Non-alcoholic liver cirrhosis & $61(1.45)$ & Hepatic coma & $51(0.64)$ \\
\hline
\end{tabular}


Table 3. Utilization of hospitalization and emergency department services in the last year of life of decedents with dementia and decedents with cancer

\begin{tabular}{|c|c|c|c|c|}
\hline & $\begin{array}{c}\text { Dementia } \\
(n=908)\end{array}$ & $\begin{array}{c}\text { Cancer } \\
(n=1816)\end{array}$ & $\overline{\text { p-value }}$ & $\begin{array}{c}\text { Adjusted odds } \\
\text { ratio }^{\dagger} \\
(95 \% \mathrm{CI})\end{array}$ \\
\hline Patients visited ED, n (\%) & $456(50.22)$ & $977(53.80)$ & 0.0778 & $0.87(0.73-1.02)$ \\
\hline \multicolumn{5}{|l|}{$\begin{array}{l}\text { Frequency of ED visits per } \\
\text { person, } \mathrm{n}(\%)\end{array}$} \\
\hline$<3$ times & $287(62.94)$ & $613(62.74)$ & 0.9431 & 1.00 (ref.) \\
\hline$\geq 3$ times & $169(37.06)$ & $364(37.26)$ & & $0.90(0.70-1.15)$ \\
\hline $\begin{array}{l}\text { Patients had acute hospital } \\
\text { care, } \mathrm{n}(\%)\end{array}$ & $748(82.38)$ & $1471(81.00)$ & 0.3834 & $1.14(0.91-1.41)$ \\
\hline \multicolumn{5}{|l|}{$\begin{array}{l}\text { Frequency of } \\
\text { hospitalization per person, } \mathrm{n} \\
(\%)\end{array}$} \\
\hline$<3$ times & $307(41.04)$ & $1047(47.72)$ & 0.0028 & 1.00 (ref.) \\
\hline$\geq 3$ times & $441(58.96)$ & $769(52.28)$ & & $1.26(1.04-1.52)$ \\
\hline
\end{tabular}

Length of stay per person, $\mathrm{n}$ $(\%)$

$$
\begin{array}{lllll}
<30 \text { days } & 299(39.97) & 1079(49.90) & <.0001 & 1.00(\text { ref. }) \\
>=30 \text { days } & 449(60.03) & 737(50.10) & 1.40(1.16-1.69)
\end{array}
$$

Patients had ICU admission, $\mathrm{n} \quad 538(59.25) \quad 519(28.58) \quad<.0001 \quad 3.45(2.90-4.11)$ $(\%)$

Frequency of ICU admissi on per person, $\mathrm{n}(\%)$

$$
\begin{array}{lllll}
<2 \text { times } & 352(65.43) & 419(80.73) & <.0001 & 1.00(\text { ref. }) \\
\geq 2 \text { times } & 186(34.57) & 100(19.27) & 2.01(1.49-2.70)
\end{array}
$$

Length of stay per person, $\mathrm{n}(\%)$

$$
\begin{array}{llll}
<\text { days } \quad 376(69.89) & 430(82.85) \quad<.0001 \quad 1.00(\text { ref. })
\end{array}
$$




$$
\geq 3 \text { days }
$$

162(30.11) 89(17.15)

$1.92(1.41-2.61)$

Doctors in charge of patients'

care during hospitalization

$\begin{array}{lrrrr}\text { Neurologists, } \mathrm{n}(\%) & 88(9.69) & 37(2.04) & <.0001 & 4.24(2.81-6.39) \\ \text { Internal medicine physician } & 451(49.67) & 657(36.18) & <.0001 & 1.85(1.56-2.19) \\ \mathrm{s}, \mathrm{n}(\%) & & & & \end{array}$

$\begin{array}{lllll}\text { Surgeons }^{\ddagger}, \mathrm{n}(\%) & 129(14.21) & 322(17.73) & 0.0197 & 0.81(0.64-1.03)\end{array}$

Orthopedic surgeons, $\mathrm{n} \quad 47(5.18) \quad 43(2.37) \quad<.0001 \quad 2.46(1.59-3.81)$ $(\%)$

$\mathrm{CI}=$ confidence interval; $\mathrm{ED}=$ emergency department; $\mathrm{ICU}=$ intensive care unit.

${ }^{\dagger}$ Adjusted for confounding covariates, including comorbidities.

‡ Excluding orthopedic surgeons. 
Table 4. Analysis of use of life-sustaining treatments in the last year of life between patients with dementia and patients with cancer

\begin{tabular}{|c|c|c|c|c|}
\hline & $\begin{array}{c}\text { Dementia } \\
(n=908)\end{array}$ & $\begin{array}{c}\text { Cancer } \\
(n=1816)\end{array}$ & p-value & $\begin{array}{c}\text { Adjusted odds } \\
\text { ratio }^{\dagger} \\
(95 \% \text { CI }) \\
\end{array}$ \\
\hline Enteral tube insertion ${ }^{\ddagger}, \mathrm{n}(\%)$ & $659(72.58)$ & $878(48.35)$ & $<.0001$ & $2.63(2.20-3.14)$ \\
\hline Tube feeding, $\mathrm{n}(\%)$ & $612(67.40)$ & $741(40.80)$ & $<.0001$ & $2.75(2.31-3.27)$ \\
\hline Blood transfusion $^{\S}, \mathrm{n}(\%)$ & $524(57.71)$ & $979(53.91)$ & 0.0601 & $1.11(0.94-1.32)$ \\
\hline \multicolumn{5}{|l|}{ Respiratory therapies } \\
\hline $\begin{array}{l}\text { Mechanical ventilation and } \\
\text { HFOV } \rrbracket, n(\%)\end{array}$ & $558(61.45)$ & $481(26.49)$ & $<.0001$ & $4.11(3.45-4.91)$ \\
\hline $\begin{array}{l}\text { Noninvasive positive pressure } \\
\text { ventilators", n (\%) }\end{array}$ & $90(9.91)$ & $138(7.60)$ & 0.0399 & $1.28(0.95-1.71)$ \\
\hline $\begin{array}{l}\text { Endotracheal intubation, } \mathrm{n} \\
(\%)\end{array}$ & $536(59.03)$ & $435(23.95)$ & $<.0001$ & $4.23(3.54-5.05)$ \\
\hline Tracheostomy, n (\%) & $89(9.80)$ & $55(3.03)$ & $<.0001$ & $3.23(2.25-4.64)$ \\
\hline Hemodialysis, n (\%) & $160(17.62)$ & $96(5.29)$ & $<.0001$ & $3.26(2.42-4.40)$ \\
\hline \multicolumn{5}{|l|}{$\begin{array}{l}\text { Cardiopulmonary } \\
\text { life-sustaining treatments }\end{array}$} \\
\hline $\begin{array}{l}\text { Defibrillation and Temporary } \\
\text { pacemaker, } \mathrm{n}(\%)\end{array}$ & $93(10.24)$ & $46(2.53)$ & $<.0001$ & $3.81(2.60-5.57)$ \\
\hline $\begin{array}{l}\text { Cardiopulmonary } \\
\text { resuscitation, } \mathrm{n}(\%)\end{array}$ & $308(33.92)$ & $183(10.08)$ & $<.0001$ & $4.36(3.51-5.41)$ \\
\hline \multicolumn{5}{|l|}{$\mathrm{CI}=$ confidence interval. } \\
\hline \multicolumn{5}{|c|}{${ }^{\dagger}$ Adjusted for confounding covariates, including comorbidities. } \\
\hline \multicolumn{5}{|c|}{${ }^{\ddagger}$ Included nasogastric tube insertions, gastrostomy, jejunostomy. } \\
\hline \multicolumn{5}{|c|}{$\S$ Whole blood, packed red blood cell transfusion. } \\
\hline 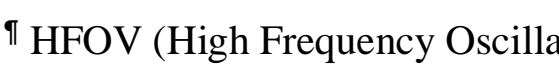 & Von & & & \\
\hline
\end{tabular}


"Included Nasal PAP (Nasal Positive Airway Pressure), CPAP (Continuous Positive Airway Pressure), Bi-PAP (Bi-level Positive Airway Pressure). 